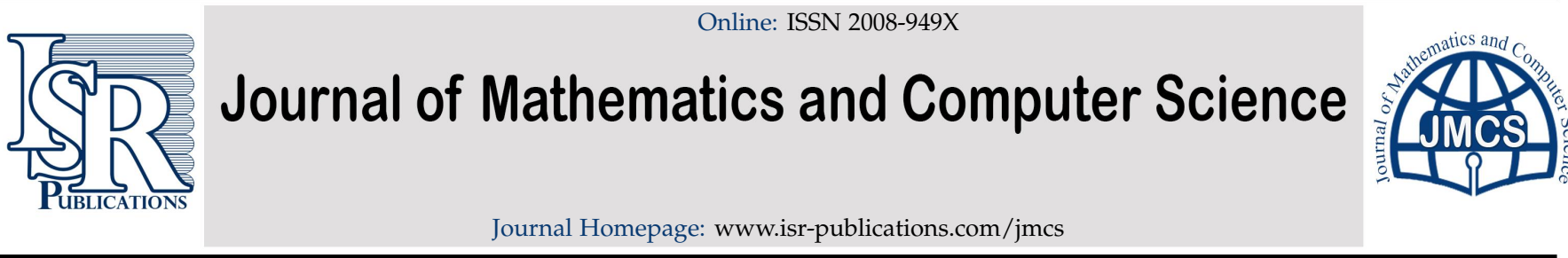

\title{
A new extended B-spline approximation technique for sec- ond order singular boundary value problems arising in physiology
}

\author{
Imtiaz Wasim ${ }^{a}$, Muhammad Abbas ${ }^{a}$, Muhammad Kashif lqbal ${ }^{b, *}$ \\ a Department of Mathematics, University of Sargodha, Sargodha, 40100, Pakistan. \\ ${ }^{b}$ Department of Mathematics, Government College University, Faisalabad, 38000, Pakistan.
}

\begin{abstract}
In this study, we have explored the approximate solution of $2^{\text {nd }}$ order singular boundary value problems (SBVP's) using extended cubic B-spline (ECBS) collocation approach. The accuracy of the numerical algorithm has been enhanced by means of a novel ECBS approximation for $2^{\text {nd }}$ order derivative. To endorse our claim, few test examples have been considered and the experimental results are compared with the already existing methods. It is observed that the proposed technique is more accurate and efficient in comparison to the existing techniques on the topic.
\end{abstract}

Keywords: Singular boundary value problems, extended B-spline functions, quasi-linearization technique, extended B-spline collocation method.

2010 MSC: 34B15, 34B16, 74H15, 65L10, 65L11.

(C)2019 All rights reserved.

\section{Introduction}

SBVPs crop up in mathematical modeling of several real life phenomena, for instance, electrohydrodynamics, chemical reactions, thermal explosions, nuclear physics, atomic structures and hydro-dynamics. They also appear in the study of some topics in physiology which include heat supply in the human head, the process of oxygen diffusion and the growth of some tumor types $[7,9,19,20]$. In this work, we have considered the following class of SBVPs with singularity at $x=0$

$$
v^{\prime \prime}(x)+\left(k_{1}+\frac{k_{2}}{x}\right) v^{\prime}(x)=\psi(x, v), 0 \leqslant x \leqslant 1,
$$

associated with one of the following conditions

$$
\begin{cases}v^{\prime}(0)=0, & v(0)=k_{3}, \\ v^{\prime}(0)=0, & k_{3} v(1)+k_{4} v^{\prime}(1)=k_{5},\end{cases}
$$

where $k_{i}$ 's are constants and $\psi$ is a non-linear function. To obtain a unique solution to this SBVP, we suppose that $\psi_{v} \geqslant 0$ and $\psi, \psi_{v}$ are continuous on $[0,1][5]$.

\footnotetext{
*Corresponding author

Email address: kashifiqbal@gcuf .edu.pk (Muhammad Kashif Iqbal)

doi: $10.22436 /$ jmcs.019.04.06
}

Received: 2019-04-23 Revised: 2019-06-12 Accepted: 2019-06-15 
The investigation of approximate solution to boundary value problems (BVPs) has always remained of great interest for many researchers due to their vast and significant role in natural world [5, 6, 16, 18, $25,28,32]$. The authors in $[6,22,27]$ investigated the approximate solution of SBVPs by means numerical schemes based on finite difference formulation. Parand et al. [23] developed a collocation approach based on Hermite function for solving singular initial value problems (IVPs). The power series solution to SBVPs was examined in $[26,34]$ using He's variational iteration procedure. A combination of Green's identities and Adomian decomposition technique was developed and applied to a class of SBVPs by Singh and Kumar [30]. Wang et al. [33] utilized a numerical scheme based on reproducing kernel method (RKM) for $2^{\text {nd }}$ order Lane-Emden equations. Babolian et al. [3] implemented Sinc-Galerkin approach for solving non-linear SBVPs. Mohsenyzadeh et al. [21] developed a technique based on orthonormal Bernoulli polynomials for solving SBVPs. Singh et al. [31] introduced a semi numerical approach for solving SBVPs of order two. Xie et al.[35] investigated the series solution of non-linear SBVPs by an improved Differential Transformation technique (IDTM).

In the last two decades, splines have been frequently used for solving IVPs and BVPs due to their rich geometrical properties. Third degree B-spline functions of order 4 were employed in [4] for investigating the approximate solution of two point linear BVPs. Rashidinia et al. [24] studied the spline solution of SBVPs using third degree polynomial functions. Khuri and Sayfy [15] presented an innovative numerical scheme by relating B-spline collocation scheme with modified Adomian decomposition method for solving SBVPs. Abukhaled et al. [2] used B-spline functions with chebyshev polynomials (CPCBSM) for solving SBVPs. Goh et al. $[10,11]$ investigated the approximate solution of linear SBVPs with the help of quartic and extended uniform B-spline functions. The authors in [14], proposed a new quartic B-spline approximation technique for numerical investigation of third order SBVPs.

In this manuscript, we have developed a numerical algorithm for approximate solution of a class of SBVPs. The proposed scheme is based on a novel ECBS approximation for $u^{\prime \prime}(x)$ and has never been employed for this purpose before.

This work is arranged as following. Section 2 provides a brief review of typical extended B-spline functions and its related results. Section 3 furnishes a new approximation for $2^{\text {nd }}$ order spatial derivative. Section 4 illustrates the implementation of the proposed approach to SBVPs of order 2. Section 5 presents computational outcomes and discussion. The concluding remarks on this study are recorded in Section 6.

\section{Extended cubic B-spline functions}

Suppose that the interval $[0,1]$ is partitioned uniformly to generate a non-decreasing sequence of nodes $0=x_{0}, x_{1}, x_{2}, \ldots, x_{n}=1$, provided that $x_{k}=x_{0}+k h, k$ is an integer and $n h=1, n \in \mathbb{N}$. The ECBS functions are defined as [29]

$E_{k}(x)=\frac{1}{24 h^{4}} \begin{cases}4 h(1-\sigma)\left(y_{k-2}\right)^{3}+3 \sigma\left(y_{k-2}\right)^{4}, & x \in\left[x_{k-2}, x_{k-1}\right], \\ (4-\sigma) h^{4}+12 h^{3}\left(y_{k-1}\right)+6 h^{2}(2+\sigma)\left(y_{k-1}\right)^{2}-12 h\left(y_{k-1}\right)^{3}-3 \sigma\left(y_{k-1}\right)^{4}, & x \in\left[x_{k-1}, x_{k}\right], \\ (4-\sigma) h^{4}+12 h^{3}\left(y_{k+1}\right)+6 h^{2}(2+\sigma)\left(y_{k+1}\right)^{2}-12 h\left(y_{k+1}\right)^{3}-3 \sigma\left(y_{k+1}\right)^{4}, & x \in\left[x_{k}, x_{k+1}\right], \\ 4 h(1-\sigma)\left(y_{k+2}\right)^{3}+3 \sigma\left(y_{k+2}\right)^{4}, & x \in\left[x_{k+1}, x_{k+2}\right], \\ 0, & \text { otherwise. }\end{cases}$

where $y_{k-2}=x-x_{k-2}, y_{k-1}=x-x_{k-1}, y_{k+1}=x_{k+1}-x$, and $y_{k+2}=x_{k+2}-x$.

It is worth noting that ECBS functions preserve the properties of cubic B-spline (CBS) functions for $\sigma \in[-8,1]$. Suppose, there exists a unique ECBS approximation, $V(x)$, to the exact analytical solution $v(x)$ which satisfies $V\left(x_{k}\right)=v\left(x_{k}\right), \forall k=0,1,2, \ldots, n$. Mathematically, we can write

$$
V(x)=\sum_{k=-1}^{n+1} \delta_{k} E_{k}(x),
$$

where, the constant quantities, $\delta_{k}$ 's are unknowns to be calculated. Using (2.1) and (2.2), $v(x)$ as well as 
its derivatives can be approximated at the $k^{\text {th }}$ knot as

$$
\begin{aligned}
V\left(x_{k}\right) & =V_{k}=\sum_{i=k-1}^{k+1} \delta_{i} E_{i}\left(x_{k}\right)=\left(\frac{4-\sigma}{24}\right) \delta_{k-1}+\left(\frac{8+\sigma}{12}\right) \delta_{k}+\left(\frac{4-\sigma}{24}\right) \delta_{k+1}, \\
V^{\prime}\left(x_{k}\right) & =m_{k}=\sum_{i=k-1}^{k+1} \delta_{i} E_{i}^{\prime}\left(x_{k}\right)=\left(\frac{-1}{2 h}\right) \delta_{k-1}+(0) \delta_{k}+\left(\frac{1}{2 h}\right) \delta_{k+1} \\
V^{\prime \prime}\left(x_{k}\right) & =M_{k}=\sum_{i=k-1}^{k+1} \delta_{i} E_{i}^{\prime \prime}\left(x_{k}\right)=\left(\frac{2+\sigma}{2 h^{2}}\right) \delta_{k-1}+\left(\frac{-(2+\sigma)}{h^{2}}\right) \delta_{k}+\left(\frac{2+\sigma}{2 h^{2}}\right) \delta_{k+1} .
\end{aligned}
$$

Also, from (2.3)-(2.5), following equations can be derived [8].

$$
\begin{aligned}
& m_{k}=u^{\prime}\left(x_{k}\right)+\frac{\sigma}{24} h^{2} v^{\prime \prime \prime}\left(x_{k}\right)+\frac{5 \sigma^{2}-10 \sigma-16}{2880} h^{4} v^{(5)}\left(x_{k}\right)+O\left(h^{6}\right), \\
& M_{k}=\left(1+\frac{\sigma}{2}\right) v^{\prime \prime}\left(x_{k}\right)+\frac{\sigma^{2}-4}{48} h^{2} v^{(4)}\left(x_{k}\right)+O\left(h^{4}\right) .
\end{aligned}
$$

\section{New ECBS approximation for second order derivative}

Following $[13,17]$ and using (2.6), the relations for $M_{k-1}$ and $M_{k+1}$ at $x=x_{k}, k=1(1) n-1$, can be written as

$$
\begin{aligned}
M_{k-1} & =1+\frac{\sigma}{2} v^{\prime \prime}\left(x_{k-1}\right)+\frac{\sigma^{2}-4}{48} h^{2} v^{(4)}\left(x_{k-1}\right)+O\left(h^{4}\right) \\
& =\frac{2+\sigma}{2} v^{\prime \prime}\left(x_{k}\right)-\frac{2+\sigma}{2} v^{\prime \prime \prime}\left(x_{k}\right)+\frac{\sigma^{2}+12 \sigma+20}{48} h^{2} v^{(4)}\left(x_{k}\right)-\frac{(2+\sigma)^{2}}{48} h^{3} v^{(5)}\left(x_{k}\right)+O\left(h^{4}\right), \\
M_{k+1} & =\frac{2+\sigma}{2} v^{\prime \prime}\left(x_{k}\right)+\frac{2+\sigma}{2} v^{\prime \prime \prime}\left(x_{k}\right)+\frac{\sigma^{2}+12 \sigma+20}{48} h^{2} v^{(4)}\left(x_{k}\right)+\frac{(2+\sigma)^{2}}{48} h^{3} v^{(5)}\left(x_{k}\right)+O\left(h^{4}\right) .
\end{aligned}
$$

To obtain a new approximation $\hat{M}_{k}$ to $v^{\prime \prime}(x)$, suppose the following linear combination

$$
\hat{M}_{k}=c_{1} M_{k}+c_{2} M_{k-1}+c_{3} M_{k+1} \text {. }
$$

The parameters $c_{1}, c_{2}$, and $c_{3}$ are picked to obtain the highest error order of $\hat{M}_{k}$. From the expression (3.1), three relations can be obtained as follows:

$$
c_{1}+c_{2}+c_{3}=\frac{2}{2+\sigma}, \quad-c_{2}+c_{3}=0, \quad\left(\sigma^{2}-4\right) c_{1}+\left(\sigma^{2}+12 \sigma+20\right) c_{2}+\left(\sigma^{2}+12 \sigma+20\right) c_{3}=0 .
$$

Hence, $c_{1}=\frac{\sigma+10}{6(\sigma+2)}$ and $c_{2}=c_{3}=\frac{2-\sigma}{12(\sigma+2)}$. Using these values and (2.5) in the expression (3.1), we get

$$
\hat{M}_{k}=\frac{(2-\sigma) \delta_{k-2}+4(4+\sigma) \delta_{k-1}-6(6+\sigma) \delta_{k}+4(4+\sigma) \delta_{k+1}+(2-\sigma) \delta_{k+2}}{24 h^{2}} .
$$

At $x=x_{0}$, we assume

$$
\hat{M}_{0}=c_{0} M_{0}+c_{1} M_{1}+c_{2} M_{2}+c_{3} M_{3}
$$

where

$$
\begin{aligned}
& M_{1}=\frac{2+\sigma}{2} v^{\prime \prime}\left(x_{0}\right)+\frac{2+\sigma}{2} h v^{\prime \prime \prime}\left(x_{0}\right)+\frac{(2+\sigma)(10+\sigma)}{48} h^{2} v^{(4)}\left(x_{0}\right)+\frac{(2+\sigma)^{2}}{48} h^{3} v^{(5)}\left(x_{0}\right)+O\left(h^{4}\right), \\
& M_{2}=\frac{2+\sigma}{2} v^{\prime \prime}\left(x_{0}\right)+(2+\sigma) h v^{\prime \prime \prime}\left(x_{0}\right)+\frac{(2+\sigma)(46+\sigma)}{48} h^{2} v^{(4)}\left(x_{0}\right)+\frac{(2+\sigma)(14+\sigma)}{24} h^{3} v^{(5)}\left(x_{0}\right)+O\left(h^{4}\right),
\end{aligned}
$$




$$
M_{3}=\frac{2+\sigma}{2} v^{\prime \prime}\left(x_{0}\right)+\frac{3(2+\sigma)}{2} h v^{\prime \prime \prime}\left(x_{0}\right)+\frac{(2+\sigma)(106+\sigma)}{48} h^{2} v^{(4)}\left(x_{0}\right)+\frac{(2+\sigma)(34+\sigma)}{16} h^{3} v^{(5)}\left(x_{0}\right)+O\left(h^{4}\right) .
$$

Again selecting the parameters $c_{0}, c_{1}, c_{2}$, and $c_{3}$ to achieve the maximum error order, the relation (3.3) generates the four expressions as follows

$$
\begin{aligned}
& \mathrm{c}_{0}+\mathrm{c}_{1}+\mathrm{c}_{2}+\mathrm{c}_{3}=1 \text {, } \\
& c_{1}+2 c_{2}+3 c_{3}=0 \text {, } \\
& (\sigma-2) \mathfrak{c}_{0}+(\sigma+10) \mathfrak{c}_{1}+(\sigma+46) \mathfrak{c}_{2}+(\sigma+106) \mathfrak{c}_{3}=0, \\
& (\sigma+2) c_{1}+2(\sigma+14) c_{2}+3(\sigma+34) c_{3}=0 .
\end{aligned}
$$

Hence, $c_{0}=\frac{14-\sigma}{6(2+\sigma)}, c_{1}=\frac{5(\sigma-2)}{12(2+\sigma)}, c_{2}=\frac{2-\sigma}{3(2+\sigma)}$, and $c_{3}=\frac{\sigma-2}{12(2+\sigma)}$, the expression (3.3) takes the following form

$$
\hat{M}_{0}=\frac{2(14-\sigma) \delta_{-1}+3(3 \sigma-22) \delta_{0}+8(7-2 \sigma) \delta_{1}+14(\sigma-2) \delta_{2}+6(2-\sigma) \delta_{3}+(\sigma-2) \delta_{4}}{24 h^{2}} .
$$

By adopting similar procedure, the approximation at $x=x_{n}$ can be expressed as

$$
\hat{M}_{n}=\frac{(\sigma-2) \delta_{n-4}+6(2-\sigma) \delta_{n-3}+14(\sigma-2) \delta_{n-2}+8(7-2 \sigma) \delta_{n-1}+3(3 \sigma-22) \delta_{n}+2(14-\sigma) \delta_{n+1}}{24 h^{2}} .
$$

\section{Description of numerical method}

By means of quasi-linearlization approach, we convert the problem (1.1) into linear form as

$$
v_{r+1}^{\prime \prime}(x)+\left(k_{1}+\frac{k_{2}}{x}\right) v_{r+1}^{\prime}(x)+X_{r}(x) v_{r+1}(x)=Y_{r}(x), \quad 0 \leqslant x \leqslant 1,
$$

where $Y_{r}(x)=\psi\left(x, v_{r}\right)-\left(\frac{\partial \psi}{\partial v}\right)_{\left(x, v_{r}\right)}, X_{r}(x)=-\left(\frac{\partial \psi}{\partial v}\right)_{\left(x, v_{r}\right)}, r=0,1,2, \ldots$ The transformed end conditions (1.2) can be given as

$$
\left\{\begin{array}{l}
v_{r+1}^{\prime}(0)=0 \\
k_{3} v_{r+1}(1)+k_{4} v_{r+1}^{\prime}(1)=k_{5}
\end{array}\right.
$$

Since, problem (1.1) contains singularity at $x=0$, we use L' Hospital's rule to get the following expression

$$
z_{1}(x) v_{r+1}^{\prime \prime}(x)+z_{2}(x) v_{r+1}^{\prime}(x)+X_{r}(x) v_{r+1}(x)=Y_{r}(x), \quad 0 \leqslant x \leqslant 1,
$$

where $z_{1}(x)=\left\{\begin{array}{ll}1+k_{2}, & \text { if } x=0, \\ 1, & \text { otherwise, }\end{array} \quad z_{2}(x)= \begin{cases}k_{1}, & \text { if } x=0, \\ k_{1}+\frac{k_{2}}{x}, & \text { otherwise. }\end{cases}\right.$

Suppose $V(x)$ be the ECBS solution to (4.2) which satisfies the initial/end conditions s.t.

$$
V(x)=\sum_{k=-1}^{n+1} \delta_{k} E_{k}(x) .
$$

For $\mathrm{k}=1: 1: \mathrm{n}-1,(4.2)$ yields

$$
z_{1}\left(x_{k}\right) V_{r+1}^{\prime \prime}\left(x_{k}\right)+z_{2}\left(x_{l}\right) V_{r+1}^{\prime}\left(x_{k}\right)+X_{r}\left(x_{k}\right) V_{r+1}\left(x_{k}\right)=Y_{r}\left(x_{k}\right)
$$

Using (2.3)-(2.4) and (3.2) in equation (4.4), we have

$$
\begin{aligned}
& z_{1}\left(x_{k}\right)\left(\frac{(2-\sigma) \delta_{k-2}+4(4+\sigma) \delta_{k-1}-6(6+\sigma) \delta_{k}+4(4+\sigma) \delta_{k+1}+(2-\sigma) \delta_{k+2}}{24 h^{2}}\right) \\
& \quad+z_{2}\left(x_{k}\right)\left(\frac{-1}{2 h} \delta_{k-1}+0 \delta_{k}+\frac{1}{2 h} \delta_{k+1}\right)+X_{r}\left(x_{k}\right)\left(\frac{4-\sigma}{24} \delta_{k-1}+\frac{8+\sigma}{12} \delta_{k}+\frac{4-\sigma}{24} \delta_{k+1}\right)=Y_{r}\left(x_{k}\right)
\end{aligned}
$$


For $k=0, n,(4.2)$ yields the following two relations

$$
\begin{aligned}
& z_{1}\left(x_{0}\right)\left(\frac{2(14-\sigma) \delta_{-1}+3(3 \sigma-22) \delta_{0}+8(7-2 \sigma) \delta_{1}+14(\sigma-2) \delta_{2}+6(2-\sigma) \delta_{3}+(\sigma-2) \delta_{4}}{24 h^{2}}\right) \\
& \quad+z_{2}\left(x_{0}\right)\left(\frac{-1}{2 h} \delta_{-1}+0 \delta_{0}+\frac{1}{2 h} \delta_{1}\right)+X_{r}\left(x_{0}\right)\left(\frac{4-\sigma}{24} \delta_{-1}+\frac{8+\sigma}{12} \delta_{0}+\frac{4-\sigma}{24} \delta_{1}\right)=Y_{r}\left(x_{0}\right), \\
& z_{1}\left(x_{n}\right)\left(\frac{(\sigma-2) \delta_{n-4}+6(2-\sigma) \delta_{n-3}+14(\sigma-2) \delta_{n-2}+8(7-2 \sigma) \delta_{n-1}+3(3 \sigma-22) \delta_{n}+2(14-\sigma) \delta_{n+1}}{24 h^{2}}\right) \\
& \quad+z_{2}\left(x_{n}\right)\left(\left(\frac{-1}{2 h}\right) \delta_{n-1}+(0) \delta_{n}+\left(\frac{1}{2 h}\right) \delta_{n+1}\right)+X_{r}\left(x_{n}\right)\left(\left(\frac{4-\sigma}{24}\right) \delta_{n-1}+\left(\frac{8+\sigma}{12}\right) \delta_{n}+\left(\frac{4-\sigma}{24}\right) \delta_{n+1}\right)=Y_{r}\left(x_{n}\right) .
\end{aligned}
$$

The end conditions (4.1) provide the following two equations

$$
\begin{aligned}
\frac{4-\sigma}{24} \delta_{-1}+\frac{8+\sigma}{12} \delta_{0}+\frac{4-\sigma}{24} \delta_{1} & =0, \\
k_{3}\left(\frac{4-\sigma}{24} \delta_{n-1}+\frac{8+\sigma}{12} \delta_{n}+\frac{4-\sigma}{24} \delta_{n+1}\right)+k_{4}\left(\frac{-1}{2 h} \delta_{n-1}+0 \delta_{n}+\frac{1}{2 h} \delta_{n+1}\right) & =k_{5} .
\end{aligned}
$$

Consequently, from (4.5)-(4.9), we get a matrix equation of the form

$$
\mathrm{L} \delta=\mathrm{m},
$$

where, $L$ is the matrix of coefficients with order $n+3, \delta=\left[\begin{array}{llll}\delta_{-1} & \delta_{0} & \delta_{1} \cdots \delta_{n+1}\end{array}\right]^{\top}$ while $m$ represents a column vector of order $n+3$. The system (4.10) is solved for $\delta_{i}$ 's and these constants are plugged into (4.3) to obtain the approximate solution. The numerical computations are executed in Mathematica 9.

\section{Numerical results and discussion}

To investigate the validity and accuracy of numerical scheme, two types of error norms, $\succeq_{\infty}, \succeq_{2}$ and experimental order of convergence (EOC) are evaluated as [1]

$$
\iota_{\infty}=\max _{k}\left|v_{k}-v_{k}\right|, \quad \iota_{2}=\sqrt{\sum_{k}\left(v_{k}-V_{k}\right)^{2}}, \quad \text { EOC }=\frac{\log \left[\frac{\succeq_{\infty}(2 n)}{\succeq_{\infty}(n)}\right]}{\log (2)},
$$

where $V_{k}$ and $v_{k}(x)$ are used for approximate and exact solutions respectively. Moreover, the numerical results are compared with CPCBSM [2], cubic basis spline method (CBSM) [4], extended cubic B-spline method (ECBM) [10], quartic B-spline method (QBSM) [11], new CBSM (NCBSM) [12], variational iteration method (VIM) [26], Green's functions associated with improved form of Adomian decomposition method (GIADM) [30], adomian decomposition method (ADM) [31], semi numerical method (SNM) [31], RKM [33], and IDTM [35].

Problem 5.1. Consider the problem (1.1) with $\mathrm{k}_{1}=0, \mathrm{k}_{2}=1, \psi=-v$ with the end conditions

$$
v^{\prime}(0)=0, v(1)=1 \text {. }
$$

The resulting equation is also known as Bessel's equation of order zero. In Table 1, the error norms obtained by the proposed method are compared with the CBSM [4], EBSM [10], and QBSM [11] using three choices of $h$. For $h=0.1,0.05,0.02$, the values $\sigma=-0.005812,-0.00066,-0.000011$ have been used respectively. It is observed that the obtained results are more accurate in comparison to other methods. The experimental order of convergence (EOC) has been reported in Table 2. The analytic solution for this problem is $v(x)=\frac{J_{0}(x)}{J_{0}(1)}$. 
Table 1: Error norms for Problem 5.1.

\begin{tabular}{ccccccccc}
\hline & \multicolumn{2}{c}{ CBSM [4] } & \multicolumn{2}{c}{ ECBSM [10] } & \multicolumn{2}{c}{ QBSM [11] } & \multicolumn{2}{c}{ Proposed method } \\
$\mathrm{h}$ & $\mathrm{E}_{\infty}$ & $\mathrm{Ł}_{2}$ & $\mathrm{E}_{\infty}$ & $\mathrm{七}_{2}$ & $\mathrm{E}_{\infty}$ & $\mathrm{七}_{2}$ & $\mathrm{E}_{\infty}$ & $\mathrm{七}_{2}$ \\
\hline 0.1 & $1.1 \mathrm{E}-04$ & $2.7 \mathrm{E}-04$ & $1.3 \mathrm{E}-05$ & $3.0 \mathrm{E}-05$ & $1.7 \mathrm{E}-06$ & $1.9 \mathrm{E}-06$ & $1.55 \mathrm{E}-08$ & $2.06 \mathrm{E}-08$ \\
0.05 & $2.8 \mathrm{E}-05$ & $9.1 \mathrm{E}-05$ & $1.2 \mathrm{E}-07$ & $4.6 \mathrm{E}-07$ & $2.0 \mathrm{E}-07$ & $2.3 \mathrm{E}-07$ & $9.34 \mathrm{E}-10$ & $2.57 \mathrm{E}-09$ \\
0.02 & $4.5 \mathrm{E}-06$ & $2.3 \mathrm{E}-05$ & $9.8 \mathrm{E}-09$ & $4.3 \mathrm{E}-08$ & $1.4 \mathrm{E}-08$ & $1.6 \mathrm{E}-08$ & $3.27 \mathrm{E}-11$ & $1.66 \mathrm{E}-10$ \\
\hline
\end{tabular}

Table 2: Experimental order of convergence (EOC) for Problem 5.1.

\begin{tabular}{ccccccc}
\hline $\mathrm{n}$ & 5 & 10 & 20 & 40 & 80 & 160 \\
\hline$\sigma$ & -0.005812 & -0.00066 & -0.00011 & -0.000052 & -0.000013 & -0.000001999 \\
$\mathrm{~L}_{\infty}$ & $2.51 \mathrm{E}-07$ & $1.55 \mathrm{E}-08$ & $9.34 \mathrm{E}-10$ & $5.18 \mathrm{E}-11$ & $3.21 \mathrm{E}-12$ & $1.58 \mathrm{E}-13$ \\
$\mathrm{EOC}$ & $\cdots$ & 4.01 & 4.05 & 4.17 & 4.01 & 4.34 \\
\hline
\end{tabular}

Problem 5.2. Setting $k_{1}=0$ and $k_{2}=2$ in (1.1) with the following end conditions

$$
v(0)=\log \frac{1}{4}, v(1)=\log \frac{1}{5} .
$$

In Table 3, the numerical results are tabulated for $h=0.05$. It is evident that the current scheme is more efficient in terms of accuracy as compared to CBSM [4], SNM [31], and ADM [31]. The absolute numerical error corresponding to $h=5,10,20,40$ has been displayed in Figure 1. The exact solution for this problem is $v(x)=\frac{1}{4+x^{k_{6}}}$ and $\psi=k_{6} x^{k_{6}-2} e^{v(x)}\left(k_{6} x^{k_{6}} e^{v(x)}-k_{2}-k_{6}+1\right)$.

\begin{tabular}{ccccc} 
Table 3: & Absolute errors for Problem 5.2 using $k_{6}=5, \sigma=-0.00102, h=0.05$ \\
\hline$x$ & CBSM [4] & ADM [31] & SNM [31] & Proposed method \\
\hline 0.0 & $1.0 \mathrm{E}-14$ & 0 & 0 & $4.0 \mathrm{E}-15$ \\
0.1 & $8.2 \mathrm{E}-06$ & $\ldots$ & $\cdots$ & $5.1 \mathrm{E}-10$ \\
0.2 & $1.1 \mathrm{E}-05$ & $8.4 \mathrm{E}-05$ & $6.0 \mathrm{E}-06$ & $5.9 \mathrm{E}-10$ \\
0.3 & $1.2 \mathrm{E}-05$ & $\cdots$ & $\cdots$ & $4.0 \mathrm{E}-10$ \\
0.4 & $1.2 \mathrm{E}-05$ & $7.4 \mathrm{E}-05$ & $2.1 \mathrm{E}-06$ & $6.1 \mathrm{E}-11$ \\
0.5 & $1.1 \mathrm{E}-05$ & $\cdots$ & $\cdots$ & $3.1 \mathrm{E}-10$ \\
0.6 & $9.2 \mathrm{E}-06$ & $3.0 \mathrm{E}-05$ & $4.8 \mathrm{E}-06$ & $6.1 \mathrm{E}-10$ \\
0.7 & $6.9 \mathrm{E}-06$ & $\cdots$ & $\cdots$ & $7.4 \mathrm{E}-10$ \\
0.8 & $4.5 \mathrm{E}-06$ & $2.0 \mathrm{E}-05$ & $9.8 \mathrm{E}-06$ & $6.7 \mathrm{E}-10$ \\
0.9 & $2.1 \mathrm{E}-06$ & $\cdots$ & $\cdots$ & $3.7 \mathrm{E}-10$ \\
1.0 & 0 & $1.6 \mathrm{E}-15$ & 0 & $4.4 \mathrm{E}-16$ \\
\hline
\end{tabular}

Problem 5.3. Consider $k_{1}=0, k_{2}=1, \psi=v^{3}(x)-3 v^{5}(x)$ in (1.1) with the initial conditions

$$
v(0)=1, v^{\prime}(0)=0 .
$$

This equation is also called Emden-Flower type equation. In Table 4, the numerical results obtained by the proposed scheme show a superior agreement with the exact analytical solution as compared to CBSM [4] and RKM [33] are listed. The exact solution for this problem is $v(x)=\frac{1}{\sqrt{1+x^{2}}}$. 


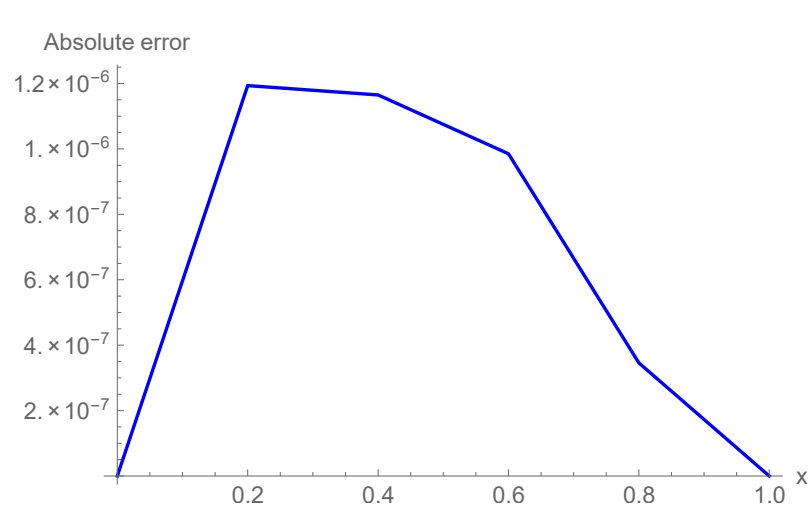

(a) $n=5, \sigma=-0.0001$

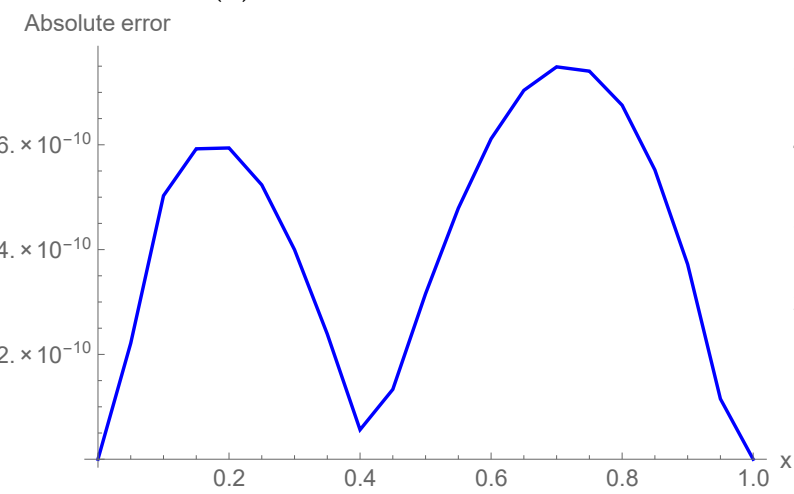

(c) $n=20, \sigma=-0.00102$

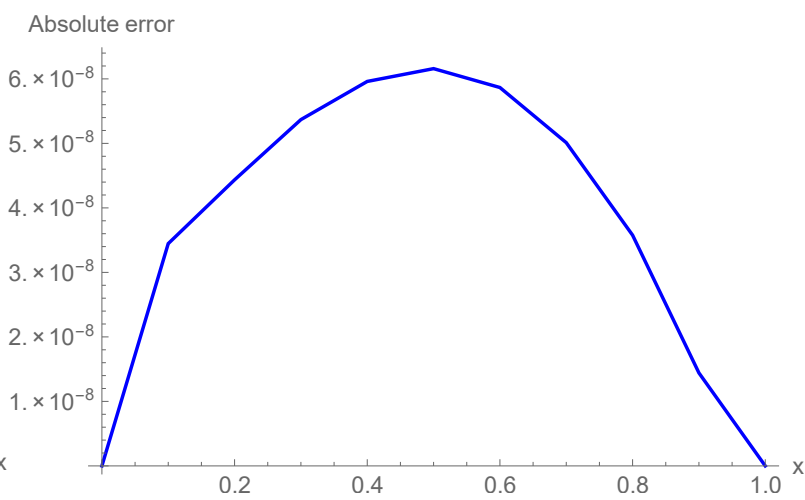

(b) $\mathrm{n}=10, \sigma=-0.0008$

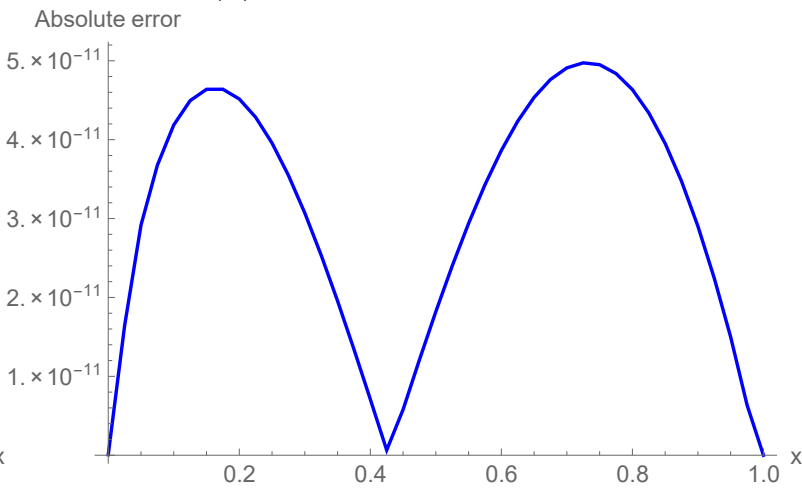

(d) $n=40, \sigma=-0.00026$

Figure 1: Absolute error for Problem 5.2.

Table 4: For Problem 5.3, absolute errors using $\mathrm{h}=0.05$ and $\sigma=-0.00744$.

\begin{tabular}{cccc}
\hline$x$ & CBSM [4] & RKM [33] & Proposed Method \\
\hline 0.0 & $6.4 \mathrm{E}-15$ & 0 & $1.5 \mathrm{E}-15$ \\
0.1 & $1.2 \mathrm{E}-06$ & $3.3 \mathrm{E}-07$ & $7.0 \mathrm{E}-08$ \\
0.2 & $4.1 \mathrm{E}-06$ & $3.5 \mathrm{E}-07$ & $6.9 \mathrm{E}-08$ \\
0.3 & $7.5 \mathrm{E}-06$ & $4.2 \mathrm{E}-07$ & $5.3 \mathrm{E}-08$ \\
0.4 & $1.0 \mathrm{E}-05$ & $5.5 \mathrm{E}-07$ & $3.5 \mathrm{E}-08$ \\
0.5 & $1.1 \mathrm{E}-05$ & $7.6 \mathrm{E}-07$ & $2.0 \mathrm{E}-08$ \\
0.6 & $9.6 \mathrm{E}-06$ & $1.0 \mathrm{E}-06$ & $7.5 \mathrm{E}-09$ \\
0.7 & $6.4 \mathrm{E}-06$ & $1.3 \mathrm{E}-06$ & $6.8 \mathrm{E}-09$ \\
0.8 & $1.6 \mathrm{E}-06$ & $1.8 \mathrm{E}-06$ & $2.4 \mathrm{E}-08$ \\
0.9 & $4.0 \mathrm{E}-06$ & $2.4 \mathrm{E}-06$ & $4.6 \mathrm{E}-08$ \\
1.0 & $1.0 \mathrm{E}-05$ & $3.7 \mathrm{E}-06$ & $6.9 \mathrm{E}-08$ \\
\hline
\end{tabular}

Problem 5.4. Consider $k_{1}=0, k_{2}=2, \psi=-v^{5}(x)$ in (1.1) associated with the end conditions

$$
v^{\prime}(0)=0, v(1)=\frac{\sqrt{3}}{2} \text {. }
$$

In Table 5, the numerical results computed by proposed scheme are compare with existing techniques on the topic. It can be observed that the proposed scheme is more accurate and powerful as compared to NCBSM [12], VIM [26], GIADM [30], and IDTM [35]. Figure 2 demonstrates the absolute error corresponding to four different choices of grid size. The exact solution for this test problem is $v(x)=\frac{1}{\sqrt{1+\frac{x^{2}}{3}}}$. 
Table 5: For Problem 5.4, absolute errors setting $h=0.05$ and $\sigma=-0.002059$.

\begin{tabular}{cccccc}
\hline$x$ & VIM [26] & GIADM [30] & IDTM [35] & NCBSM [12] & Proposed method \\
\hline 0.0 & $6.3 \mathrm{E}-03$ & $3.2 \mathrm{E}-03$ & $1.7 \mathrm{E}-04$ & $9.4 \mathrm{E}-08$ & $1.7 \mathrm{E}-09$ \\
0.1 & $6.3 \mathrm{E}-03$ & $3.1 \mathrm{E}-03$ & $1.7 \mathrm{E}-04$ & $9.0 \mathrm{E}-08$ & $7.5 \mathrm{E}-11$ \\
0.2 & $6.1 \mathrm{E}-03$ & $3.0 \mathrm{E}-03$ & $1.6 \mathrm{E}-04$ & $8.6 \mathrm{E}-08$ & $8.3 \mathrm{E}-11$ \\
0.3 & $5.9 \mathrm{E}-03$ & $2.6 \mathrm{E}-03$ & $1.6 \mathrm{E}-04$ & $7.9 \mathrm{E}-08$ & $1.8 \mathrm{E}-10$ \\
0.4 & $5.5 \mathrm{E}-03$ & $2.2 \mathrm{E}-03$ & $1.5 \mathrm{E}-04$ & $7.0 \mathrm{E}-08$ & $3.5 \mathrm{E}-10$ \\
0.5 & $5.1 \mathrm{E}-03$ & $1.8 \mathrm{E}-03$ & $1.4 \mathrm{E}-04$ & $5.9 \mathrm{E}-08$ & $5.9 \mathrm{E}-10$ \\
0.6 & $4.5 \mathrm{E}-03$ & $1.4 \mathrm{E}-03$ & $1.2 \mathrm{E}-04$ & $4.7 \mathrm{E}-08$ & $8.4 \mathrm{E}-10$ \\
0.7 & $3.8 \mathrm{E}-03$ & $9.8 \mathrm{E}-04$ & $1.1 \mathrm{E}-04$ & $3.4 \mathrm{E}-08$ & $1.1 \mathrm{E}-09$ \\
0.8 & $2.9 \mathrm{E}-03$ & $6.1 \mathrm{E}-04$ & $9.5 \mathrm{E}-05$ & $2.2 \mathrm{E}-08$ & $1.0 \mathrm{E}-09$ \\
0.9 & $1.6 \mathrm{E}-03$ & $2.8 \mathrm{E}-04$ & $6.8 \mathrm{E}-05$ & $1.0 \mathrm{E}-08$ & $8.4 \mathrm{E}-10$ \\
1.0 & $1.0 \mathrm{E}-10$ & $3.5 \mathrm{E}-08$ & 0 & $1.1 \mathrm{E}-16$ & $1.1 \mathrm{E}-16$ \\
\hline
\end{tabular}

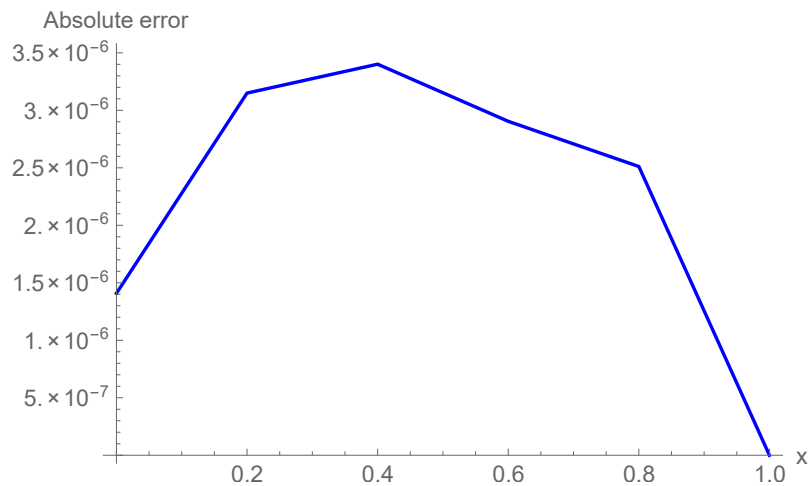

(a) $n=5, \sigma=-0.0034$

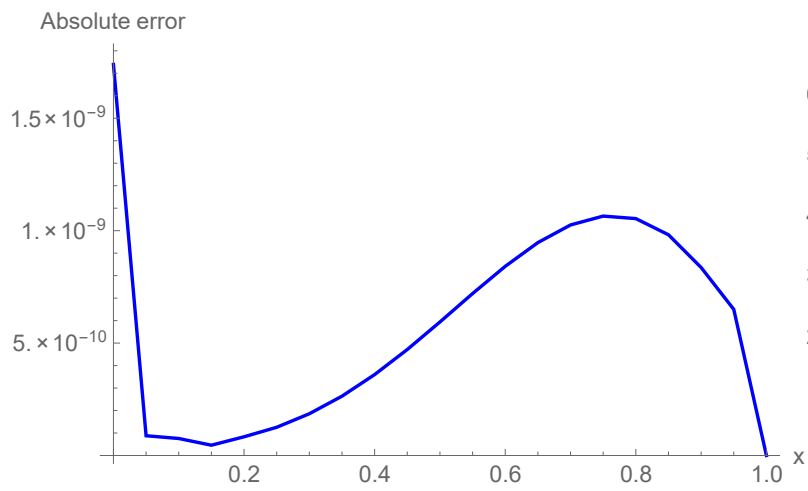

(c) $\mathrm{n}=20, \sigma=-0.002059$

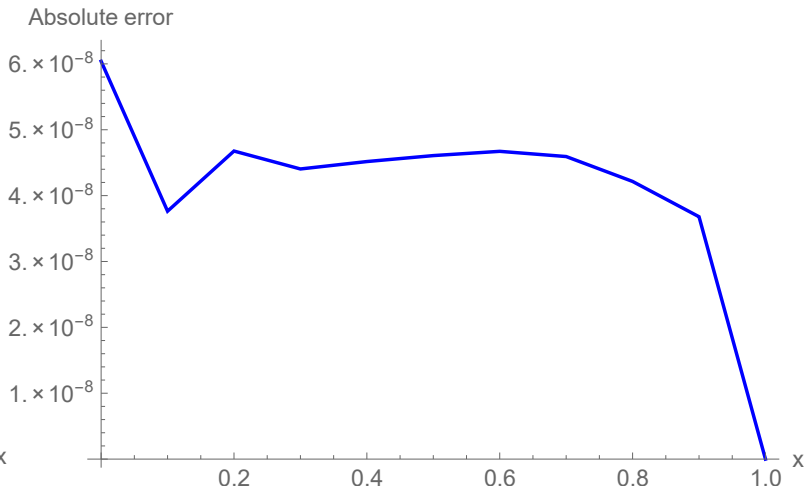

(b) $n=10, \sigma=-0.0083$

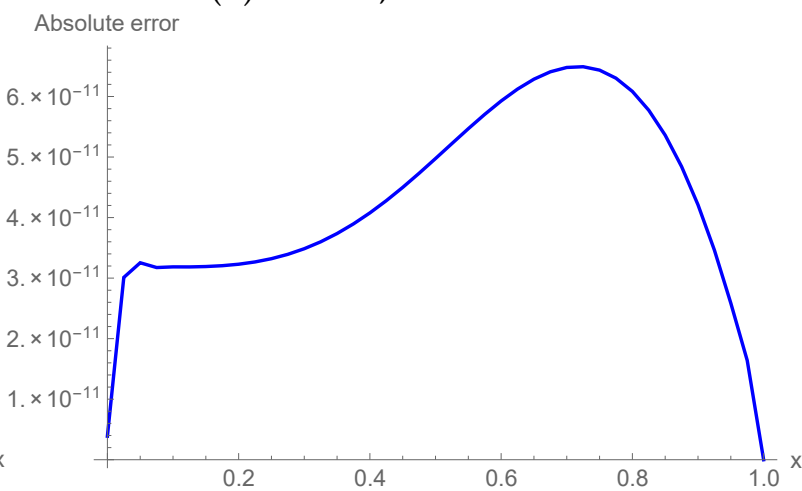

(d) $n=40, \sigma=-0.00052$

Figure 2: Absolute error for Problem 5.4.

\section{Conclusions}

In this work, the approximate solution of a class of $2^{\text {nd }}$ order singular boundary value problems is studied by employing extended B-spline collocation technique. The accuracy of the proposed numerical scheme has been enhanced by means of a new approximation for $2^{\text {nd }}$ order derivative. The computational results are compared with those obtained by CPCBSM [2], CBSM [4], ECBSM [10], QBSM [11], NCBSM [12], VIM [26], GIADM [30], ADM [31], SNM [31], RKM [33], and IDTM [35]. It is concluded that the presented algorithm has very simple implementation and produces more accurate outcomes as compared to existing numerical techniques. 


\section{References}

[1] M. Abbas, A. A. Majid, A. I. M. Ismail, A. Rashid, The application of cubic trigonometric B-spline to the numerical solution of the hyperbolic problems, Appl. Math. Comput., 239 (2014), 74-88. 5

[2] M. Abukhaled, S. A. Khuri, A. Sayfy, A numerical approach for solving a class of singular boundary value problems arising in physiology, Int. J. Numer. Anal. Model., 8 (2011), 353-363. 1, 5, 6

[3] E. Babolian, A. Iftikhari, A. Saadarmandi, A Sinc-Galerkin technique for the numerical solution of a class of singular boundary value problems, Comput. Appl. Math., 34 (2015), 45-63. 1

[4] N. Caglar, H. Caglar, B-spline solution of singular boundary value problems, Appl. Math. Comput., 182 (2006), 15091513. $1,5,5,1,5,3,5,4,6$

[5] Z. D. Cen, Numerical study for a class of singular two-point boundary value problems using Greens functions, Appl. Math. Comput., 183 (2006), 10-16. 1

[6] M. M. Chawala, R. Subramanian, H. L. Sathi, A fourth order method for a singular two-point boundary value problem, BIT Numer. Math., 28 (1988), 88-97. 1

[7] U. Flesch, The distribution of heat sources in the human head: a theoretical consideration, J. Theor. Biol., 54 (1975), 285-287. 1

[8] D. J. Fyfe, The use of cubic splines in the solution of two-point boundary value problems, Comput. J., 12 (1969/70), 188-192. 2

[9] J. B. Garner, R. Shivaji, Diffusion problems with a mixed nonlinear boundary condition, J. Math. Anal. Appl., 148 (1990), 422-430. 1

[10] J. Goh, A. A. Majid, A. I. M. Ismail, Extended cubic uniform B-spline for a class of singular boundary value problems, Sci. Asia, 37 (2011), 79-82. 1, 5, 5, 1, 6

[11] J. Goh, A. A. Majid, A. I. M. Ismail, A quartic B-spline for second-order singular boundary value problems, Comput. Math. Appl., 64 (2012), 115-120. 1, 5, 5, 1, 6

[12] M. K. Iqbal, M. Abbas, N. Khalid, New Cubic B-spline Approximation for Solving Non-linear Singular Boundary Value Problems Arising in Physiology, Comm. Math. Appl., 9 (2018), 377-392. 5, 5, 5, 6

[13] M. K. Iqbal, M. Abbas, I. Wasim, New cubic B-spline approximation for solving third order EmdenFlower type equations, Appl. Math. Comput., 331 (2018), 319-333. 3

[14] M. K. Iqbal, M. Abbas, B. Zafar, New Quartic B-Spline Approximation for Numerical Solution of Third Order Singular Boundary Value Problems, Punjab. Univ. J. Math., 51 (2019), 43-59. 1

[15] S. A. Khuri, A. Sayfy, A novel approach for the solution of a class of singular boundary value problems arising in physiology, Math. Comp. Modelling, 52 (2010), 626-636. 1

[16] M. Kumar, Y. Gupta, Methods for solving singular boundary value problems using splines: a review, J. Appl. Math. Comput., 32 (2010), 265-278. 1

[17] F. G. Lang, X. P. Xu, A new cubic B-spline method for approximating the solution of a class of nonlinear second-order boundary value problem with two dependent variables, Sci. Asia, 40 (2014), 444-450. 3

[18] P. M. Lima, M. P. Carpentier, Iterative methods for a singular boundary-value problem, J. Comput. Appl. Math., 111 (1999), 173-186. 1

[19] S. H. Lin, Oxygen diffusion in a spherical cell with nonlinear oxygen uptake kinetics, J. Theor. Biol., 60 (1976), $449-457$. 1

[20] D. L. S. McElwain, A re-examination of oxygen diffusion in a spherical cell with Michaelis-Menten oxygen uptake kinetics, J. Theor. Biol., 71 (1978), 255-263. 1

[21] M. Mohsenyzadeh, K. Maleknejad, R. Ezzati, A numerical approach for the solution of a class of singular boundary value problems arising in physiology, Adv. Difference Equ., 2015 (2015), 10 pages. 1

[22] R. K. Pandey, A. K. Singh, On the convergence of a finite difference method for a class of singular boundary value problems arising in physiology, J. Comput. Appl. Math., 166 (2004), 553-564. 1

[23] K. Parand, M. Dehghan, A. Z. Rezaei, S. M. Ghaderi, An approximation algorithm for the solution of the nonlinear Lane-Emden type equations arising in astrophysics using Hermite functions collocation method, Comput. Phys. Comm., 181 (2010), 1096-1108. 1

[24] J. Rashidinia, R. Mohammadi, R. Jalilian, The numerical solution of non-linear singular boundary value problems arising in physiology, Appl. Math. Comput., 185 (2007), 360-367. 1

[25] A. S. V. Ravi Kanth, Cubic spline polynomial for non-linear singular two-point boundary value problems, Appl. Math. Comput., 189 (2007), 2017-2022. 1

[26] A. S. V. Ravi Kanth, K. Aruna, He's variational iteration method for treating nonlinear singular boundary value problems, Comput. Math. Appl., 60 (2010), 821-829. 1, 5, 5, 5, 6

[27] R. D. Russell, L. F. Shampine, Numerical methods for singular boundary value problems, SIAM J. Numer. Anal., 12 (1975), 13-36. 1

[28] L. F. Shampine, Singular boundary value problems for ODEs, Appl. Math. Comput., 138 (2003), 99-112. 1

[29] S. Sharifi, J. Rashidinia, Numerical solution of hyperbolic telegraph equation by cubic B-spline collocation method, Appl. Math. Comput., 281 (2016), 28-38. 2

[30] R. Singh, J. Kumar, An efficient numerical technique for the solution of nonlinear singular boundary value problems, Comput. Phys. Commun., 185 (2014), 1282-1289. 1, 5, 5, 5, 6 
[31] R. Singh, A.-M. Wazwaz, J. Kumar, An efficient semi-numerical technique for solving nonlinear singular boundary value problems arising in various physical models, Int. J. Comput. Math., 93 (2016), 1330-1346. 1, 5, 5, 3, 6

[32] W. Y. Wang, M. G. Cui, B. Han, A new method for solving a class of singular twopoint boundary value problems, Appl. Math. Comput., 206 (2008), 721-727. 1

[33] Y. L. Wang, H. Yu, F. G. Tan, S. G. Li, Using an effective numerical method for solving a class of Lane-Emden equations, Abstr. Appl. Anal., 2014 (2014), 8 pages. 1, 5, 5, 4, 6

[34] A. M. Wazwaz, The variational iteration method for solving nonlinear singular boundary value problems arising in various physical models, Commun. Nonlinear Sci. Numer. Simul., 16 (2011), 3881-3886. 1

[35] L.-J. Xie, C.-L. Zhou, S. Xu, An effective numerical method to solve a class of nonlinear singular boundary value problems using improved differential transform method, Springer Plus, 5 (2016), 21 pages. 1, 5, 5, 5, 6 